\title{
Vortex with the Formation of Electricity Generation and System Modelling
}

\author{
Sezgin Ersoy
}

\begin{abstract}
Water resources are one of the natural sources for producing electricity on earth. Hydroelectric power plants are constructed for producing electricity through these resources. These power plants convert potential energy of the water to kinetic energy by means of using their turbines in order to produce electricity.

Water flowing through the wings of these turbines causes it to rotate hence generates electricity. Our goal is to use the same amount of water to generate a vortex, in other words allow flow of water so that constant force is applied at each wing and thus increase the rotation force.

Our system is a prototype of a hydroelectric power plant but the rotation of the turbine is designed in a way to allow vortex generation.
\end{abstract}

Index Terms-Water vortex, electricity generation, System modeling.

\section{INTRODUCTION}

Increase in human population increases the need for energy. Accordingly the energy consumption increases rapidly. This situation has become a global problem.

Countries act irrational and uncontrolled production of energy. On the other hand developing awareness of saving has being important in personal use.

Some studies are to obtain the electric vortex. Siegan investigated on numerical analysis of the influence of Distance between osculating part and surface Water due to ocean current vortex power. There was electricity discuss about the influence of distance between osculating part and surface water (d) due to maximum amplitude of osculating part using software ANSYS 11.0 computational fluid dynamic with finite element method. Analyzing shows that minimum distance of $d$ is $3 \mathrm{D}$, surface water is not significant more to influence the maximum amplitude of osculating part [1].

Hakim study on a study on the effect of distances between oscillating parts in vortex-associated power generation approach [2].

Perman study effect of free surface on the oscillating part concerning to the performance lies vortex power. As a result; the deeper the location of the oscillating part, the greater amplitude average can be produced and vice versa when tested at a greater speed in the same depth then the resulting amplitude will be smaller. This is due to many reasons, one of which is the holder stability who may not have been perfect [3]. Huda study examined the effect of vortex on plant yield

Manuscript received July 9, 2013; revised September 16, 2013.

Sezgin Ersoy is with Marmara University Technical Education Facility Mechatronics Department, Turkey. (e-mail: sersoy@marmara.edu.tr) osculating size [4]. Zotlöterer has constructed a low-head power plant that makes use of the kinetic energy inherent in an artificially induced vortex. The water's vortex energy is collected by a slow moving, large-surface water wheel, making the power station transparent to fish - there are no large pressure differences built up, as happens in normal turbines. It can see at Fig. 1. [5].

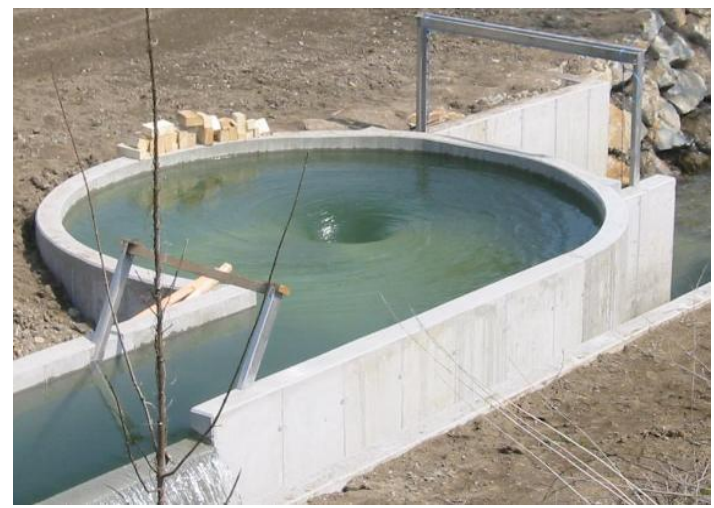

Fig. 1. Special turbine collecting vortex energy [5]

Bernidnas and friends study on a new concept in generation of clean and renewable energy from fluid flow (Fig. 2). Their work finished successfully and they indicate a lot of advantage in this project. [6]

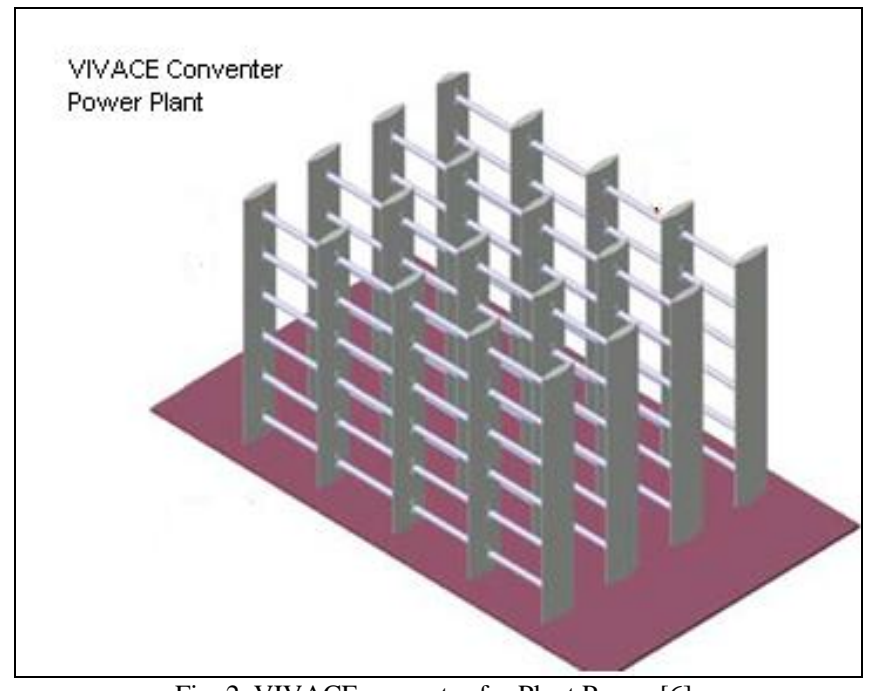

Fig. 2. VIVACE convertor for Plant Power [6]

Objective of this study that the alternative to limited water resources to achieve maximum efficiency.

\section{MATERIALS AND METHOD}

Works according to the principle of open-loop system generated. Provides resource moves required direction and 
the formation of the water vortex. However, due to the necessity of the high cost of the first example of a study had been carried out on the principle of a closed system. In our system, Flowing water has accumulated in two water tanks and vortex formed. The water that is in two tanks releases and reaches to vortex pool. And then the vortex begins to form in this pool. The mechanical fins are returning in the pool via vortex and moves the rotating coil fins are attached. As a result of this process consists of power generation. This system works as turbine.

In our system, a dynamo is used for electricity production. This dynamo was placed on the vortex chamber. In this system, two neodymium magnets, a shaft, the reservoir lid, 4 coils and 1 bearing are located. This system works on the principle of the dynamo (Fig. 3).

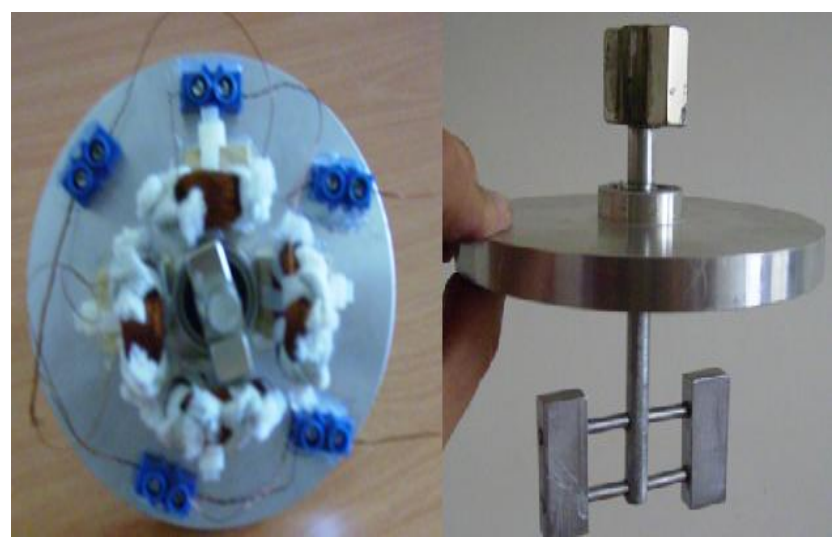

Fig. 3. Coil and Magnet component.

\section{A. Building of System Design}

This system is a prototype of hydroelectric power plants due to the fact that the principles and ways of working However, each element designed specially and strength values were calculated to the design of the system's components are as follows:

\section{1) Cylinder water tanks}

Volume of storage was calculated as follows: $V=\pi . r . r . h$. Each of cylinder volumes is $33.969 \mathrm{dm}^{2}$. The cylinders (Fig. 4.) are positioned on a pedestal (Fig. 5). Fig. 6 indicate that Chamber of mechanical drawing. Payload is determined to be 646,016 by the stress analysis. Solid Edge is used for this analysis.

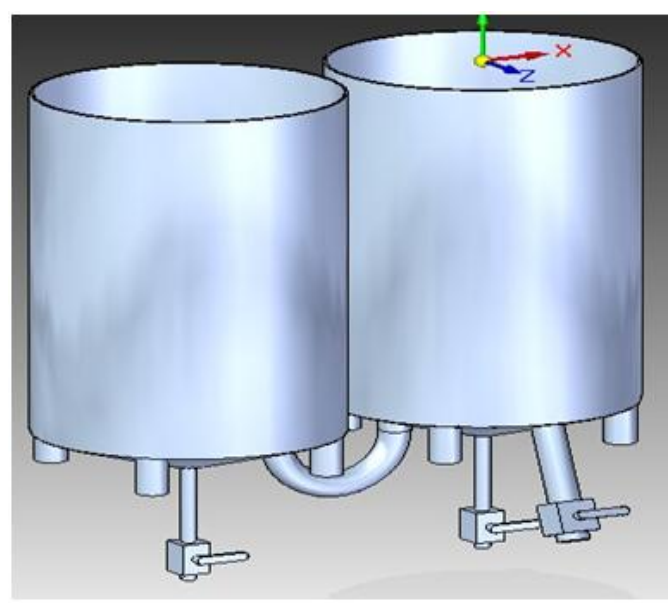

Fig. 4. Solid Model for cylinder tanks and carrier cantilever.

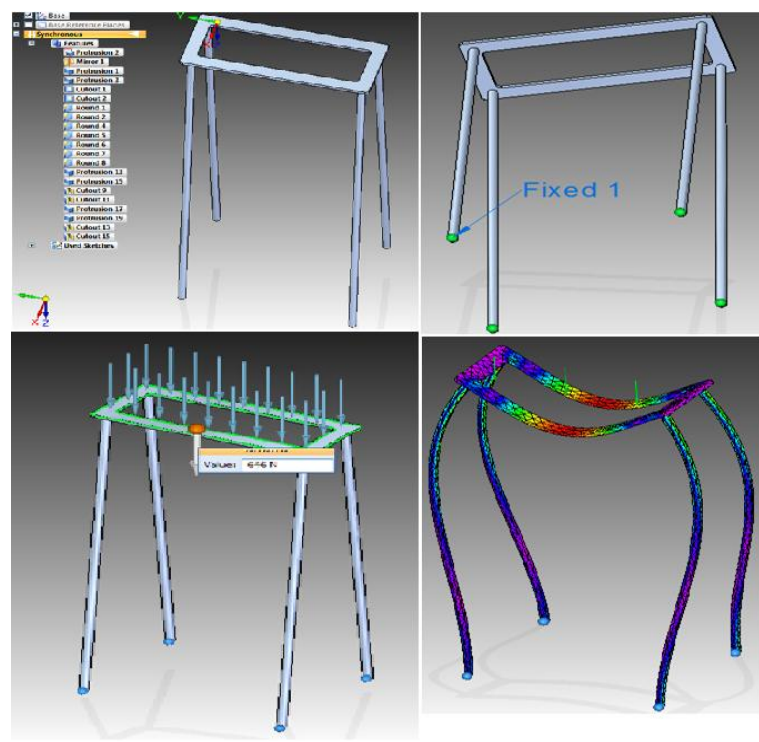

Fig. 5. Solid Model for cylinder tanks and carrier cantilever.

\section{2) Chamber of vortex formation}

The potential energy of water into the reservoir with a certain income and vortex is created. The occurrence of vortex is related with water transfer. The come is designed for faster and stronger flow of water. Angles opposite each other on the water pipes occurs turbulence in the flow of water reservoir. The water has to speed and pipe diameters should be lower.

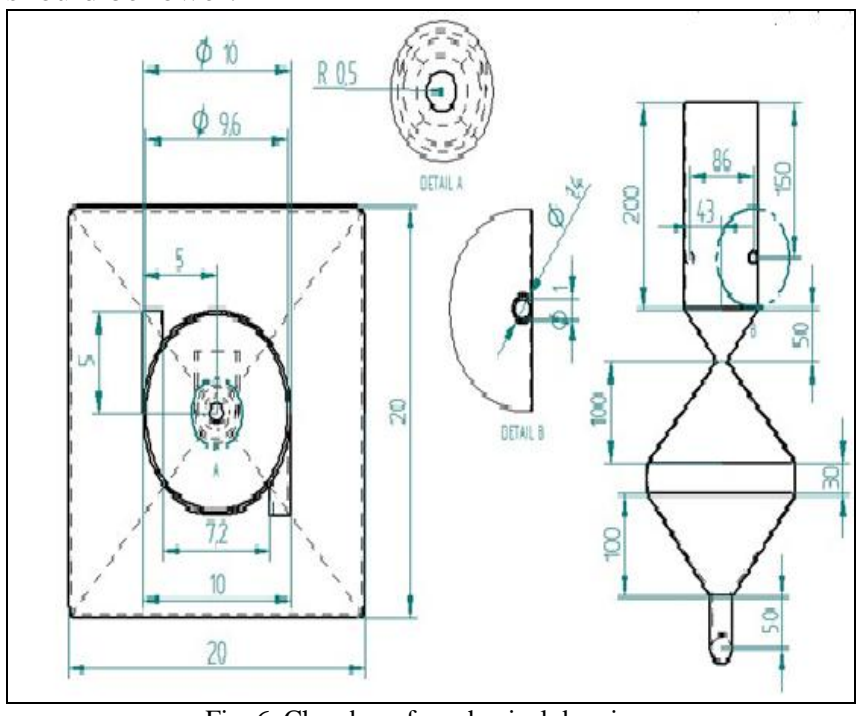

Fig. 6. Chamber of mechanical drawing.

In the system potential energy, kinetic energy and flow is calculated as the dynamic effects.

$$
(\mathrm{Ep}=(65.92) .(9.8) .(0.8)=516.81 \text { joule })
$$

\section{3) Wings, Shaft and Cover}

Mechanical energy is the sum of potential energy and kinetic energy. It is the energy associated with the motion and position of an object. The law of conservation of mechanical energy states that in an isolated system that is only subject to conservative forces the mechanical energy is constant. If an object is moved in the opposite direction of a conservative net force, the potential energy will increase and if the speed (not the velocity) of the object is changed, the kinetic energy of the object is changed as well. In all real systems, however, non-conservative forces, like frictional forces, will be present, 
but often they are of negligible values and the mechanical energy's being constant can therefore be a useful approximation. In elastic collisions, the mechanical energy is conserved but in inelastic collisions, some mechanical energy is converted into heat. The equivalence between lost mechanical energy and an increase in temperature was discovered by James Prescott Joule.

Many modern devices, such as the electric motor or the steam engine, are used today to convert mechanical energy into other forms of energy, e.g. electrical energy, or to convert other forms of energy, like heat, into mechanical energy [7].

Taking all this theoretical status, we considered; formation of vortex motion moves the flaps. Transmits the movement of the bearing shaft attached to it here. Sealing is important for bearings. Dynamo power structure was created to get the rotating shaft. Thus, by varying the magnetic field through the coil is able to generate electrical energy. To ensure this, the location is good way to measure the coil and magnets. Faraday and Lenz laws are basic reference in our study.

Fig. 7 show that A magnet and a coil. The magnet drew near towards the coil at $\mathrm{v}$ speed. In this case there is an increase in the magnetic field to the left. $(\Delta \mathrm{B})$ This corresponds to an increase in $\mathrm{B}_{\text {induced }}$ occurs. İt is shown with by the arrow. We examined to second sample that seen that the magnetic field to the right. If the magnet coils to be removed with speed $\mathrm{v}$ is an increase in the magnetic field to the left.

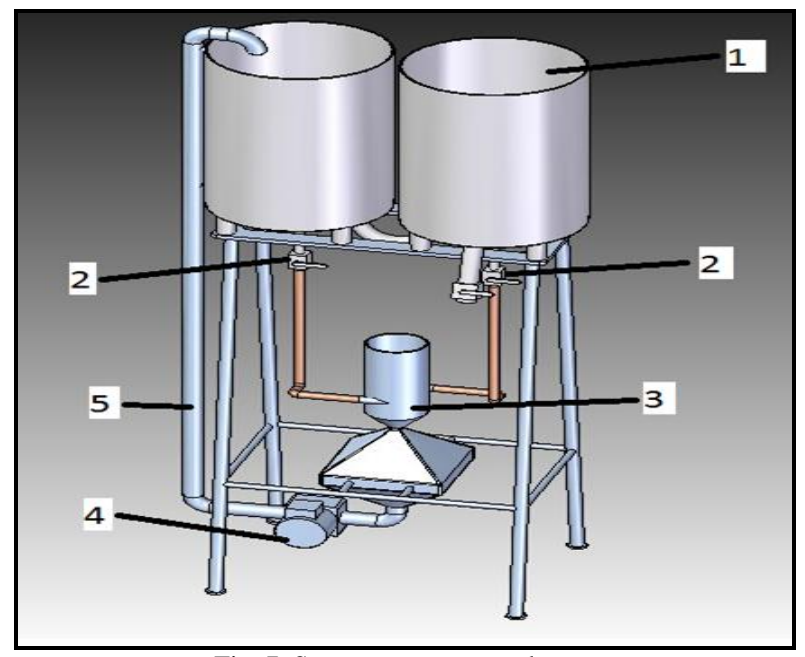

Fig. 7. System prototypes schema.

\section{4) Coil winding}

The Coils are consisted $3 \mathrm{~cm}$ in diameter and has 300 winding.

\section{5) Vortex}

Vortex motion is called rotational or turbulent fluid and distance from the center of this movement speed decreases. In accordance with Bernoulli's Law, the fluid pressure is low at the center and in inverse proportion to distance from the center increases.

\section{CONCLUSIONS}

In this study, our aim is not to provide high values of electricity generation. Only, renewable electricity produces the design water flow. Meanwhile, this system will use in many areas such as dam, river and sanitary installations.

Obtained in this study with a small vortex is provided at the level of about 1 volt of electricity generation. Many factors had caused power loss such as fraction and used materials. These effects can be reduced to systems prepared with a better budget. This system is planned to be formed if the yield of plants is higher than the normal system of the dam that will become advantageous to use. This system can be added as an innovation in the design of hydroelectric power plants.

\section{ACKNOWLEDGMENTS}

This work has been supported by the Scientific Research Project Program of Marmara University (Project number: FEN-D-120813-0354). The author is grateful to Marmara University for their financial support.

\section{REFERENCES}

[1] A. Siagian, "Numerical analysis of the influence of distance between osculating part and surface water due to ocean current vortex electricity," Master thesis, Department of Ocean Engineering Faculty of Marine Technology Institute Technology of Sepuluh, 2011.

[2] M. A. R. Hakim, "A Study on the effect of distances between oscillating parts in vortex-associated power generation: an experiment approach," Master thesis, Department of Ocean Engineering Faculty of Marine Technology Institute Technology of Sepuluh, 2011.

[3] D. W. Permana, "Study effect of free surface on the oscillating part concerning to the performance lies vortex power: physical test phenomenological," Master Thesis, Department of Ocean Engineering Faculty of Marine Technology Institute Technology of Sepuluh, 2011.

[4] L. Huda, "A study on the effect of dimensional oscillating part of vortex power plant using physical test," Master Thesis, Department of Ocean Engineering Faculty of Marine Technology Institute Technology of Sepuluh, 2011.

[5] Water vortex drives power plant (2007) [Online]. Available: http://blog.hasslberger.com/2007/06/water_vortex_drives_power_pla n.html

[6] M. M. Bernitsas, K. Raghavan, Y. Ben-Simon, and E. M. H. Garcia, "VIVACE (Vortex Induced Vibration for Aquatic Clean Energy): a new concept in generation of clean and renewable energy from fluid flow," in Proc. of OMAE2006, ASME Transactions, 2008 25th International OMAE Conf. Hamburg, Germany, 2006.

[7] Mechanical_energy [Online]. http://en.wikipedia.org/wiki/Mechanical_energy

Available:

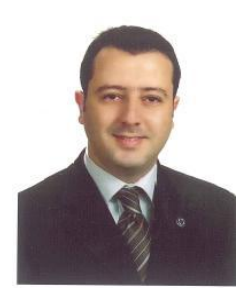

Sezgin Ersoy was born in Istanbul Turkey in 1977. $\mathrm{He}$ was graduated from Automotive Education. He has double master degree where Technology Education and Mechatronics. He finished doctoral thesis in Marmara University. He work at Marmara University as Lecturer. His interest Mechatronics, polymer science and education science. He wrote book is publish in Germany and has two book chapter publish in USA. In addition that he has 12 artical published in SCI index. Dr. Sezgin Ersoy is member some social and scientific organization, He has 4 national awards. He is married and has a son. 